\title{
Color Classifier for Symptomatic Soybean Seeds Using Image Processing
}

\author{
Irfan S. Ahmad, Research Associate, John F. Reid, Professor, Marvin R. Paulsen, Professor, Department of \\ Agricultural Engineering, and James B. Sinclair, Professor, Department of Crop Sciences, University of Illinois at \\ Urbana-Champaign, Urbana 61801
}

\begin{abstract}
Ahmad, I. S., Reid, J. F., Paulsen, M. R., and Sinclair, J. B. 1999. Color classifier for symptomatic soybean seeds using image processing. Plant Dis. 83:320-327.

Symptoms associated with fungal damage, viral diseases, and immature soybean (Glycine max) seeds were characterized using image processing techniques. A Red, Green, Blue (RGB) color feature-based multivariate decision model discriminated between asymptomatic and symptomatic seeds for inspection and grading. The color analysis showed distinct color differences between the asymptomatic and symptomatic seeds. A model comprising six color features including averages, minimums, and variances for RGB pixel values was developed for describing the seed symptoms. The color analysis showed that color alone did not adequately describe some of the differences among symptoms. Overall classification accuracy of $88 \%$ was achieved using a linear discriminant function with unequal priors for asymptomatic and symptomatic seeds with highest probability of occurrence. Individual classification accuracies were asymptomatic 97\%, Alternaria spp. 30\%, Cercospora spp. 83\%, Fusarium spp. 62\%, green immature seeds $91 \%$, Phomopsis spp. 45\%, soybean mosaic potyvirus (black) $81 \%$, and soybean mosaic potyvirus (brown) $87 \%$. The classifier performance was independent of the year the seed was sampled. The study was successful in developing a color classifier and a knowledge domain based on color for future development of intelligent automated grain grading systems.
\end{abstract}

Additional keywords: feature space, grain quality, machine vision

The midwestern United States is a major marketer of soybean (Glycine max (L.) Merr.) seeds. The grading of soybean seeds is an important task in determining product quality, price, and marketability. At present, grading is conducted manually, and oil and protein contents are not routinely identified. Grading standards of the Grain Inspection Packers Stockyards AgencyFederal Grain Inspection Service (GIPSAFGIS) of the United States Department of Agriculture (USDA) report a damage factor that includes damage due to fungal activity but does not distinguish between severely damaged and superficially damaged soybean seeds. Soybean seed diseases can cause significant losses in productivity and marketability. For example, during the years 1970, 1972-77, and 1984-87, Phomopsis spp. seed decay caused more than $2 \%$ loss for Illinois growers (17). At this threshold, grain buyers discount the price paid for soybeans, and loss to the disease has a direct economic effect.

Corresponding author: Irfan S. Ahmad
E-mail: i-ahmad1@uiuc.edu

Accepted for publication 25 November 1998.

Publication no. D-1999-0119-03R

(C) 1999 The American Phytopathological Society
Manual grading is a tedious task and requires good visual skills. Inspectors base their judgment on experience and intuition; hence, a logical explanation is not always forthcoming. Training and experience involve an inherently complex mental process that has to be transformed into a logical decision. Fuzzy inference techniques have potential to model the logical relationships (3). Furthermore, a method such as machine vision sensing for automating the grading process could bring increased speed and objectivity and provides an opportunity for improvements in marketing.

Some of the features used to describe the visual characteristics of the seed include color, morphology, and texture. Asymptomatic seeds are almost round, compact, and have a smooth seed coat and beige color. Color of the hilum is dependent on the cultivar. The size and shape of the seed may depend on the location of the seed in the pod. Green immature seed varies in color from whitish green to dark green and is a result of immaturity due to frost.

Morphological properties of diseased soybean seeds often are changed in a characteristic manner (18). Seed affected by Alternaria spp. are small, shriveled, with light to dark brown lesions covering parts of the seeds. Cercospora spp. seed damage is variable in size and color, ranging from light to dark brown, purple, or gray. Seed coats typically become pink to red due to infection by Fusarium spp. The North Central Regional Committee report showed production losses due to Cercospora spp. to be 25.25 metric tons and for Fusarium spp. to be 50.53 metric tons (8). Seeds severely affected by Phomopsis spp. are shriveled and elongated, and seed coats are cracked and chalky (17). Oil and flour extracted from Phomopsis-infected seed can be unmarketable (16). Yellow-seeded cultivars infected with soybean mosaic potyvirus produce seeds that are mottled brown or black, depending on the hilum color, with a smooth coat (13). The mottling usually appears as a "bleeding" of the hilum pigment over the seed coat.

Differences in symptoms among pathogen-damaged soybean seeds may be distinguished based on color. Other distinguishing features with potential include morphology and texture (1). Color image analysis is based on a set of values represented in several models to identify color variations. These representations may use Red, Green, Blue (RGB), which can be transformed to: hue, saturation, intensity (HSI); hue, saturation, value (HSV); cyan, magenta, yellow (CMY); red, green, blue (rgb) chromaticity coordinates; and y-axis, in-phase, quadrature (YIQ) used in broadcast television. For accurate color specification, three qualitatively different parameters must be measured. Sometimes features can be segmented by transforming RGB coordinates to another coordinate space (15). As an example, chromaticity values mask out the effects of intensity changes (2).

Earlier machine vision studies used various gray-level and color representations for grain quality evaluation with varying success $(5,10,14,19)$. Some of these studies included both corn and soybean seeds; therefore, direct comparative analysis was not appropriate. Chromaticity coordinates were used in an earlier study on symptomatic soybean seeds, but they did not include black discoloration due to soybean mosaic potyvirus (5). Quantification of color using machine vision systems requires careful consideration of all these factors to provide meaningful measurements (2).

The objective of this study was to identify features using color analysis, which 
best described symptomatic soybean seeds, and develop a color classifier for discriminating between asymptomatic and symptomatic seeds, and their types.

\section{MATERIALS AND METHODS}

A $90 \mathrm{MHz}$ Pentium computer with a Comet image processing board (Matrox Electronic Systems, Ltd., Dorval, Quebec) was used to collect images from a Sony Model XC-711 CCD RGB 768(H) $\times$ 493(V) color camera (Sony Semiconductor Company of America, San Jose, CA). The charged coupled device (CCD) camera had three primary color filters (RGB) in a striped filter configuration on a single chip to achieve high-fidelity color reproduction. The camera had an RGB signal output in addition to a composite video signal output. Gain control was set to 2.0 ( 0 db position) and $\gamma$ correction to 1.0 ("on" position). The camera was fitted with an Invaritar lens P/N 59 LGA 450 (Melles Griot, Inc., Auburn, MA) that had a large diameter and low distortion. For preliminary testing and evaluation of techniques and procedures, a script-based language native to Matrox Inspector was used.

A cylindrical light chamber internally coated with a flat white enamel paint was used to provide diffuse front lighting to the soybean seed samples (4). Two 75W quartz halogen lamps were set at 9.0 VDC supplied by a regulated DC power source. The two lamps were angled $45^{\circ}$ upward in opposing directions toward the chamber walls $20 \mathrm{~cm}$ from the center of the chamber at either side.
We analyzed 645 seeds comprising asymptomatic seeds and six types of seed surface symptoms from crop years 1989 through 1994. The damage types were green immature seeds (gis) or symptoms caused by Cercospora kikuchii (purple seed stain) (cer), Alternaria alternata (alt), Fusarium spp. (fus), and Phomopsis spp. (pho) (18). An additional category representing brown (smvbr) symptoms of soybean mosaic potyvirus (smv) for color and texture analysis was used and treated separately from black symptoms associated with the same virus (smvbk). The damage types were classified into fungal damage, viral infection, and seed immaturity, according to GIPSA-FGIS standards (9), by the Department of Crop Sciences and the Illinois Farm Bureau Crop Services Division technicians (Table 1).

Color analysis of the seeds was based on an RGB histogram of pixel values tested. RGB representation is important in digital image processing due to its wide use in digital imaging hardware, such as color cameras and monitors. An International Commission on Illumination (CIE: Commission Internationale de l'Eclairage) designated specific wavelengths to the primary colors: Red $700 \mathrm{~nm}$, Green $546.1 \mathrm{~nm}$, and Blue $435.8 \mathrm{~nm}$ (6).

The color calibration was carried out using spherical red, green, blue, black, gray, and white disks from Labsphere (Labsphere, Inc., Sutton, NH). Each disk was placed in the lighting chamber, and RGB values were recorded for each disk at three different locations. The average of

Table 1. Soybean seed samples analyzed across years and the quantity of training and test samples

\begin{tabular}{llccc}
\hline Seed type (symptoms) & Years & Seeds (no.) & Training sample & Test sample \\
\hline Asymptomatic & $1989-94$ & 135 & 60 & 60 \\
Cercospora spp. & $1989-93$ & 100 & 41 & 41 \\
Green immature & $1989-94$ & 100 & 44 & 44 \\
$\begin{array}{l}\text { Soybean mosaic potyvirus } \\
\text { (black) }\end{array}$ & $1989-94$ & 100 & 42 & 42 \\
Alternaria spp. & 1991 & 60 & 21 & 21 \\
Fusarium spp. & 1991 & 60 & 23 & 23 \\
$\begin{array}{l}\text { Phomopsis spp. } \\
\text { Soybean mosaic potyvirus }\end{array}$ & 1991 & 61 & 23 & 23 \\
$\quad$ (brown) & $1991-94$ & 30 & 14 & 14 \\
Total & & 645 & 268 & 268 \\
\hline
\end{tabular}

the three locations was used as a benchmark and saved in an ASCII file for future data runs. For each data run, values were compared and identical lighting conditions were ensured. At the start of each data run, the system was switched on for at least 15 min before data collection to ensure stabilization of the system.

An algorithm was developed to compute average pixel values for each of the RGB components separately for each seed sample. Color features of seeds were extracted interactively using a custom-developed analysis program. This algorithm was developed using Microsoft C Compiler (Microsoft Corporation, Redmond, WA) and the Matrox Image Series color board. On-board functions were used from the Matrox imaging library.

Many seeds had two or more different region types including symptomatic and asymptomatic. RGB histograms were collected on regions of each seed and used to evaluate seed color and sensitivity and response of the sensor to color variations. Histograms were extracted from three different regions: background, asymptomatic areas, and symptomatic areas. Asymptomatic seeds had only asymptomatic regions. Seeds with symptoms associated with cer and smv had three regions, while alt and fus had two. Green immature seeds and pho had variations unique to their surface characteristics, which may have taken the form of two or more symptomatic regions of varying intensity and surface texture. For each damage type, overlapping regional information was recorded due to the masking of local variations in color when the image was treated at a global level.

Images of soybeans seeds were captured and processed by carrying out multilevel thresholding to remove the background. Initially, the object (seed) was segmented from the dark background, then if symptomatic, the asymptomatic and symptomatic areas were identified using RGB values. Varying regions of interest (roi) were evaluated: the whole seed, 63 by 63 , 30 by 30 , and 15 by 15 pixels. For each seed, a 30 by 30 pixel roi was identified from the symptomatic region and RGB

Table 2. Red, Green, and Blue color feature values based on 15 by 15 pixel region of interest

\begin{tabular}{|c|c|c|c|c|c|c|c|c|}
\hline \multirow[b]{2}{*}{ Feature $^{\mathrm{b}}$} & \multicolumn{8}{|c|}{ Seed types ${ }^{\mathrm{a}}$} \\
\hline & asy & cer & gis & $s m v b k$ & alt & fus & pho & smvbr \\
\hline ravg & 187 & 124 & 161 & 77 & 146 & 155 & 156 & 149 \\
\hline gavg & 146 & 90 & 135 & 55 & 109 & 119 & 123 & 103 \\
\hline bavg & 103 & 71 & 89 & 40 & 72 & 82 & 89 & 65 \\
\hline rmin & 136 & 48 & 76 & 30 & 91 & 96 & 59 & 87 \\
\hline gmin & 96 & 38 & 65 & 24 & 62 & 65 & 46 & 50 \\
\hline bmin & 64 & 32 & 40 & 18 & 32 & 44 & 30 & 34 \\
\hline rvar & 10.9 & 83.2 & 15.1 & 238.2 & 48.4 & 24.9 & 89.9 & 76.3 \\
\hline gvar & 11.0 & 77.8 & 12.1 & 139.7 & 53.7 & 26.0 & 89.2 & 92.4 \\
\hline bvar & 14.9 & 37.5 & 12.1 & 57.0 & 42.1 & 21.4 & 78.3 & 82.7 \\
\hline
\end{tabular}

${ }^{a}$ asy $=$ asymptomatic, cer $=$ Cercospora spp., gis $=$ green immature seed, smvbk = soybean mosaic potyvirus (black), alt $=$ Alternaria spp., fus $=$ Fusarium spp., pho = Phomopsis spp., smvbr = soybean mosaic potyvirus (brown).

b $r=$ Red, $g=$ Green, $b=$ Blue; $a v g=$ average on a scale of 0 to 255 ; $\min =$ minimum; $v a r=$ variance values. 
values were analyzed. It was observed that almost identical information was achieved with varying roi; therefore, to reduce the computation time, the roi was reduced to 15 by 15 pixels.

To investigate the marginal distributions, VTFIT was used for fitting probability distribution functions to sample data and to aid in visual and formal interpretation of the goodness-of-fit (7). Several variables were found to be skewed and needed transformations.

The initial RGB data set was reduced using Pearson correlation coefficients. From each of the two highly correlated features, one was selected for further evaluation (11). The final data set comprised averages (avg), minimums ( $\mathrm{min}$ ), and variances (var) for each of the RGB components. Features involving maximums, medians, and standard deviations were dropped from further evaluation, as they were highly correlated with averages and variances. The reduced data set then was evaluated using stepwise discriminant analysis for classification of seed types. The entry criteria were set to $F=3.84$ and 2.71 for entry and removal, respectively. The summary table obtained gave the significance of each discriminant function (if
$P<0.05$, the function was considered to discriminate significantly among groups). To achieve a reduction in the problem to a lower dimension, functions that were not significant were discarded. The goal of the analysis was to determine which RGB features provided the best model for highest classification accuracy.

Most of the individual features had significantly different variances for each symptomatic group. In such cases, quadratic discriminant functions, which are based on the separate covariance matrices for the groups instead of the pooled one, were used. Quadratic analysis does not

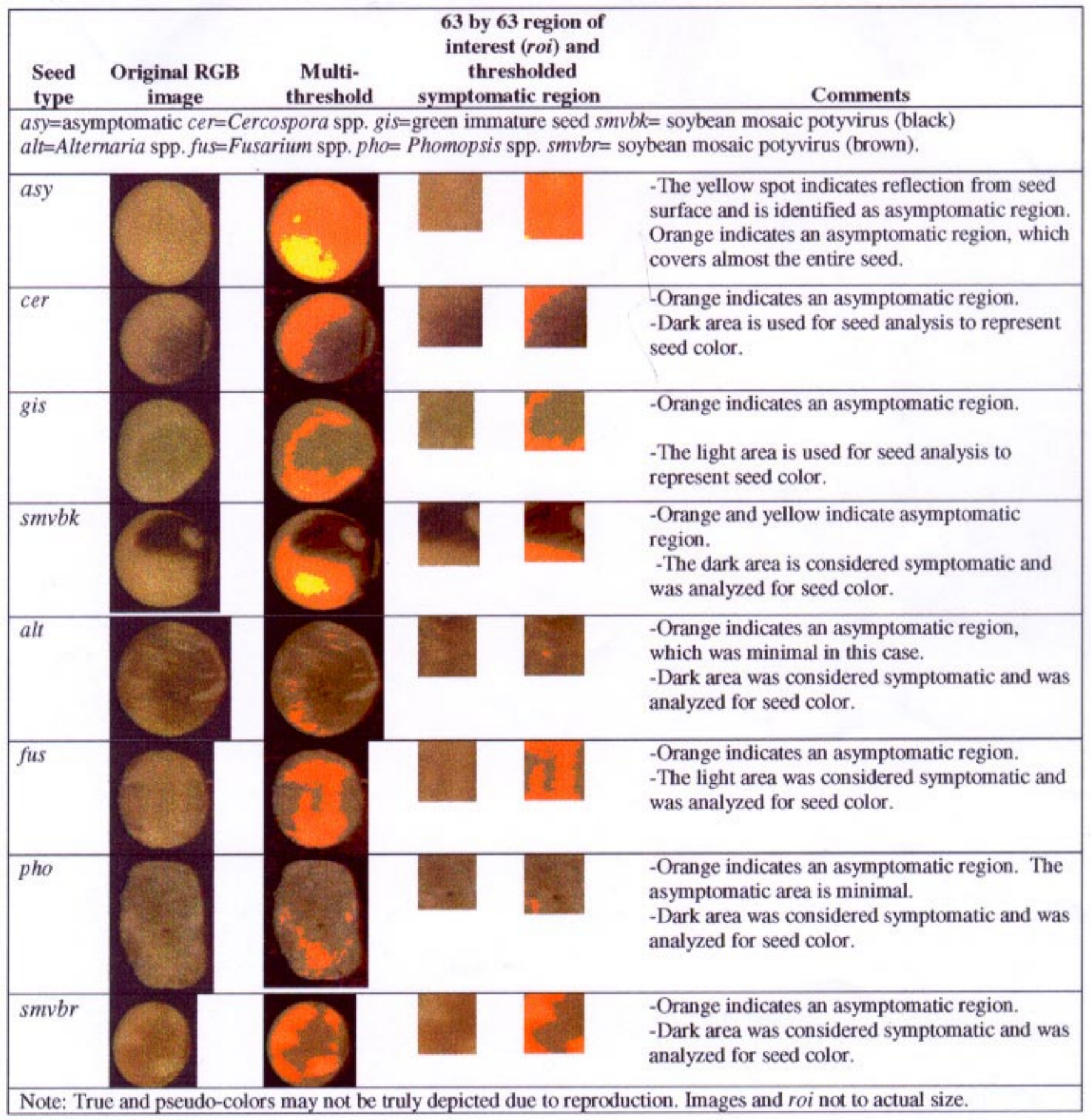

Fig. 1. Visual representation of multi-thresholding operation to separate symptomatic regions from asymptomatic regions of seeds. Asymptomatic region of the seed is shown in orange. 
perform very well for large sample sizes. However, the linear discriminant function worked well if the within-group covariance matrix was the same for each group (11). Marks and Dunn (12) showed that a linear function was satisfactory in cases where covariance matrices were not too different. Due to large sample sizes and differences between covariance matrices, both linear and quadratic discriminant analyses were performed. It was observed that most of the time, the quadratic analysis gave a more accurate classification, but not significantly better than the discriminant analysis. Due to their simplicity and ease of interpretation, linear functions were used with the understanding that optimality would be hard to achieve since there were instances when assumptions were violated.

The data were not from a random sample and did not represent the entire soybean seed population. For this reason, outside estimates of the frequency of occurrence of the diseases were used as prior probabilities of classification. Discriminant analysis was performed using equal prior and unequal (estimated) probabilities. For the aforementioned reasons, the second classification did not fit this particular data set as well; but in an application involving random samples, the second one was expected to be more accurate than the one with equal priors. Therefore, in this paper, clas- sification based on unequal priors is reported. The accuracy was dependent on the accuracy and reliability of the priors, so the results obtained have to be viewed in that context.

\section{RESULTS AND DISCUSSION}

The individual RGB color features for 645 seeds were averaged for each seed category (Table 2). These measurements were based on 15 by 15 pixel regions of interest and were reflective of asymptomatic and symptomatic regions. Asymptomatic seeds had the highest average R, G, and $B$ values due to the beige appearance of the seed. The intense black of smvbk tended to lower the average RGB values toward 0 . The largest RGB variation was found for smvbk, followed by cer and pho, but for asymptomatic and gis the variances were low. It was observed that rmin (minimum of Red component) was unique for each of the seed types (Table 2).

Multilevel thresholding was conducted to separate the background and object and the asymptomatic from symptomatic region on the seed. Original RGB images with the thresholding process for each category are shown (Fig. 1). The multithreshold column shows the asymptomatic and symptomatic regions of the seed, with pixel values below 45 set to 0 to represent background, and above 160 set to 255 to
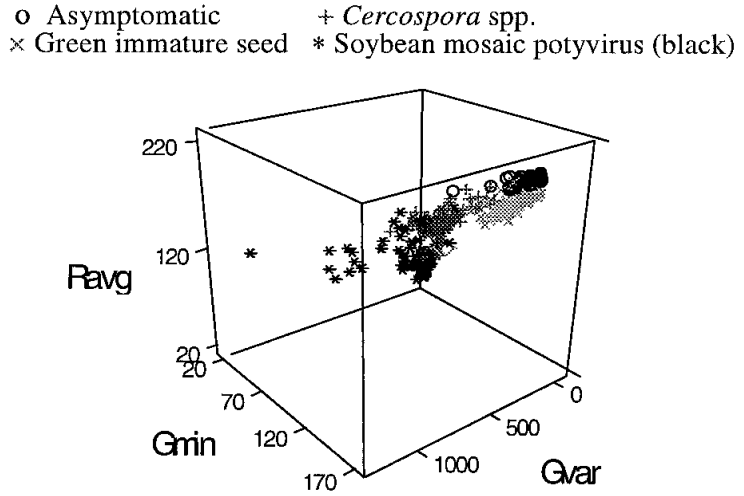

Fig. 2. Seeds of other colors in Red, Green, and Blue (RGB) feature space. represent the asymptomatic region. The pixel values 46 to 159 represented symptomatic regions of the seeds. For asymptomatic seeds, pseudo-orange color represented the asymptomatic region, with yellow considered as part of the asymptomatic region. The difference in appearance was the reflective surface of the seed. For cer and $s m v$, the asymptomatic region was a continuous area, with a distinct symptomatic region. In the case of materially damaged seeds, the asymptomatic regions varied in size and shape and were discontinuous, surrounded by symptomatic regions. This nonuniformity among materially damaged seeds made the distinction based on color more difficult compared with seeds of other colors.

Several RGB features were visualized using 2- and 3-D color feature spaces. The surface characteristics of soybean seeds, such as color, varied depending on the causal agent. Seed samples exhibited symptoms of more than one pathogen, which is normal (18).

Figure 2 shows selected features for RG components for asymptomatic and seeds of other colors individually. Each seed category is represented by distinguishable clusters, but clusters overlap. The major overlap occurs between cer and smvbk due to their frequent similar color variations. Manual observations of both seed types showed instances of cer with dark purple stains and $s m v b k$ with light to dark black surface appearance. Green immature seed is more in proximity to asy due to the Green component.

Figures 3 and 4 show 2-D plots of R and $G$ averages with distinct separation between asymptomatic and symptomatic seeds. Since the Blue component was not visualized to have distinctly separable information, it was not considered in these plots. Gis approaches asy due to its light green color (Fig. 3). Higher R and G values contributed to the beige appearance of asy.

A 3-D representation of $\mathrm{R}, \mathrm{G}$, and $\mathrm{B}$ averages with distinct separation between asymptomatic and symptomatic seeds is shown (Fig. 5A). In this representation, 15

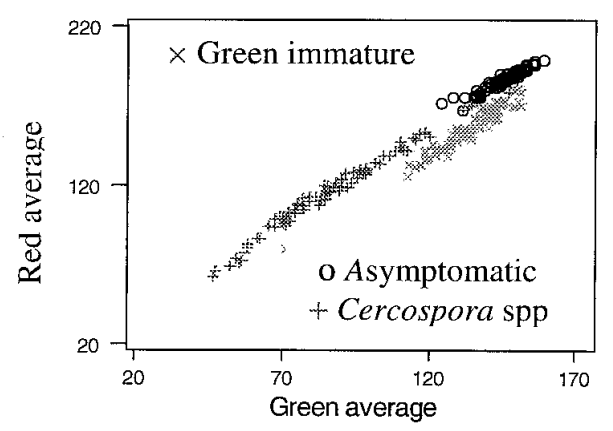

Fig. 3. Red and Green pixel value averages form three distinct clusters for asy, cer, and gis.

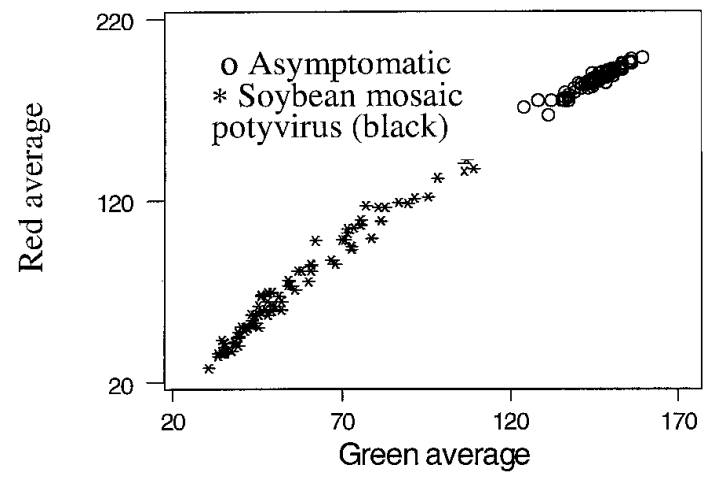

Fig. 4. Red and Green pixel value averages for asy and smvbk forming two distinct clusters. 
by 15 roi was used only on the symptomatic region of the seed located next to the hilum. Figure 6B shows only seeds of other colors with overlapping cer and smvbk clusters. Gis had some overlap with smvbr.

Color classifier. Six discriminant functions with unequal priors were computed with six variables for each of the eight seed types. The first two discriminant functions were selected because they represented the largest ratio of between-groups to withingroups sum of squares. The developed expressions are given as:

$$
\begin{aligned}
D_{\text {color } 1}= & -3.41-0.12 * \text { ravg }+0.02 * \text { rmin } \\
& +0.24 * \text { gavg }+0.03 * \text { gmin }-0.16 * \\
& \text { bavg }+0.006^{*} \text { bmin } \\
D_{\text {color } 2}= & -5.29+0.11 * \text { ravg }+0.09 * \text { rmin } \\
& -0.32 * \text { gavg }+0.01 * \text { gmin }+0.22 * \\
& \text { bavg }-0.08 * \text { bmin }
\end{aligned}
$$

The canonical correlation for a function is the square root of the between-groups to total sums of squares. The canonical discriminant functions were derived so that the pooled within-groups variance was 1 . Function 1 accounted for $58.5 \%$ of the total between-groups variability, and function 2 accounted for $29.18 \%$ variability; and the variability reduced progressively to $0.05 \%$ for function 6 (Table 3 ).

A test of the null hypothesis, that the population means of all discriminant func- tions in all groups are equal and zero, can be based on Wilks' $\lambda$. The significance level of the observed Wilks' $\lambda$ was based on $\chi^{2}$ transformation of the statistic (Table $3)$. Since observed $\alpha<0.00005$, the null hypothesis that means of the functions are equal in the eight populations can be rejected. Table 3 shows the successive removal of the functions with associated statistics. The column labeled "After $f c n$ " shows the number of the last function removed. The 0 indicates that no functions were removed, while a value of 5 indicates that the first five functions were removed. In this case, Wilks' $\lambda$ associated with function 5 after function 4 has been removed is 0.994. As this was the final remaining function, the Wilks' $\lambda$ obtained is simply the univariate value. The significance levels associated with fourth and fifth functions were 0.021 and 0.504 , respectively, showing that they did not contribute to group differences. Only the first two functions showed maximum variability; therefore, the four remaining functions (3 to 6) were not considered (Table 3).

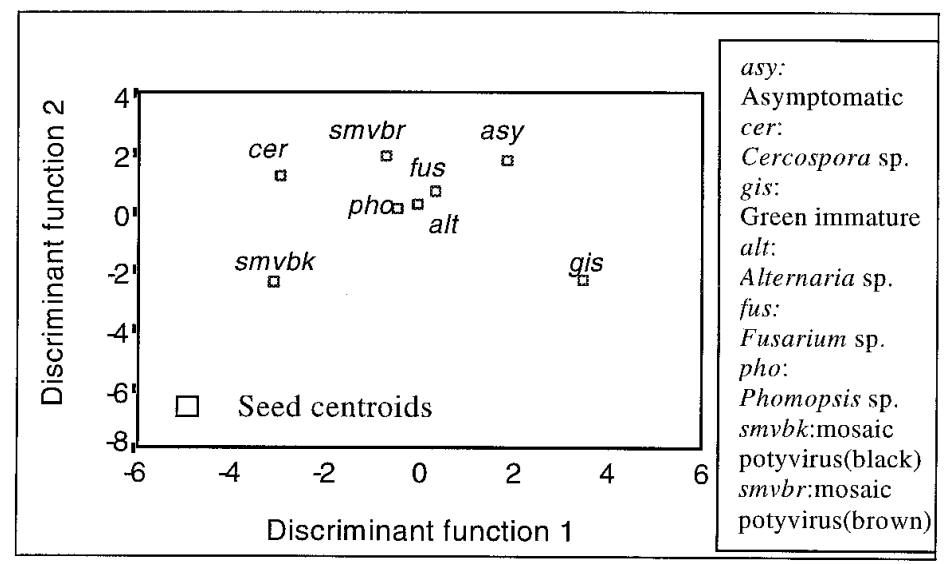

Fig. 6. Linear discriminant functions with separable seed groups with distinct centroids. asy, cer, gis, $s m v b k$, and smvbr are distinct from alt, fus, and pho.

\section{o Asymptomatic $\times$ Green immature seed}

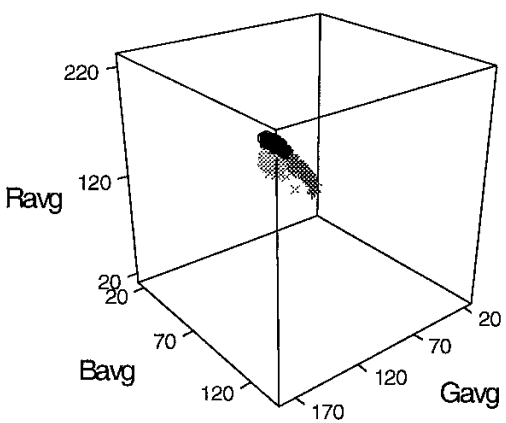

+ Cercospora spp. $x$ Green immature seed

* Soybean mosaic potyvirus (black) * (brown)

\begin{tabular}{|c|c|c|c|c|c|c|c|c|}
\hline $\operatorname{Fcn}^{\mathrm{a}}$ & Eigen value & Variance $(\%)$ & $\begin{array}{c}\text { Canonical } \\
\text { correlation }\end{array}$ & After $f c n$ & Wilks $\lambda$ & $\chi^{2}$ & df & $\sigma$ \\
\hline & & & & 0 & 0.014 & $1,105.8$ & 42 & 0.0000 \\
\hline 1 & 5.8923 & 58.50 & 0.924 & 1 & 0.097 & 603.9 & 30 & 0.0000 \\
\hline 2 & 2.9391 & 29.18 & 0.863 & 2 & 0.386 & 247.4 & 20 & 0.0000 \\
\hline 3 & 0.8845 & 8.78 & 0.685 & 3 & 0.727 & 82.7 & 12 & 0.0000 \\
\hline 4 & 0.2985 & 2.96 & 0.479 & 4 & 0.944 & 14.8 & 6 & 0.0216 \\
\hline 5 & 0.0531 & 0.53 & 0.224 & 5 & 0.994 & 1.3 & 2 & 0.5042 \\
\hline 6 & 0.0053 & 0.05 & 0.224 & & & & & \\
\hline
\end{tabular}

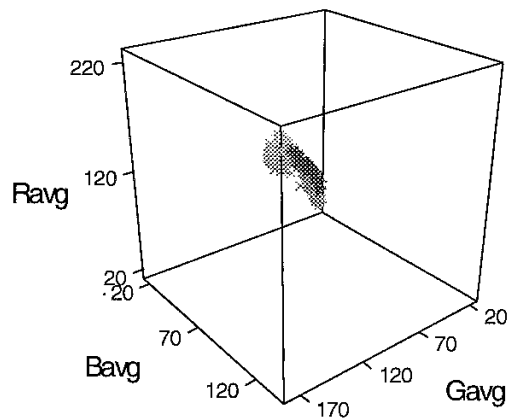

\section{A}

Fig. 5. 3-Dimensional cluster plots in Red, Green, and Blue (RGB) feature space for (A) asymptomatic, Cercospora spp., and green immature seed, and (B) Cercospora spp., green immature seed, soybean mosaic potyvirus (black), and soybean mosaic potyvirus (brown).

Table 3. Analysis of discriminant functions for classification of soybean seed categories

a The six canonical discriminant functions $(f c n)$ remaining in the analysis. 
Table 4 shows individual matrices for classification accuracy for unequal priors for the test data set. Asymptomatic seed accuracy is $98 \%$. The higher accuracy for the unequal priors can be attributed to a higher probability of occurrence of asymptomatic seed of $81.45 \%$ (1).

For cer, the highest misclassification of $7.1 \%$ occurred with asy, while some instances of misclassification are observed with asymptomatic, smvbk, alt, and pho. In the case of gis, a misclassification of $29.5 \%$ occurred with the asymptomatic category due to similar RGB variances (Table 2).

To assess the performance of the classifier without the inclusion of asymptomatic seeds, further evaluation was carried out based on the combined training and test data files (1). In the first stage, performance was evaluated by eliminating asymptomatic seeds, being the major source of misclassification from the data set. In the second stage, asymptomatic and materially damaged seeds were removed. In the third stage, asymptomatic and seeds of other colors were removed from the original data set. In the final stage, performance was evaluated based on probability of occurrence of asymptomatic and symptomatic seeds. Classification accuracy increased when only symptomatic seeds were considered (Table 5), compared with when both asymptomatic and symptomatic were considered (Table 4).

The elimination of asymptomatic and materially damaged seeds resulted in an increase in classification accuracy (Table 6). The individual accuracy for cer was $94 \%$, gis was $99 \%$, smvbk was $89 \%$, and smvbr was $97 \%$. Similarly, among the materially damaged seeds, alt had $76 \%$ and pho $82 \%$ classification accuracy (Table 7). In the case of fus, the classifier performed poorly due to lower probability of occurrence and overlapping characteristics with alt and pho. A higher individual and overall classification accuracy was achieved for seeds of other colors compared with materially damaged seeds (Tables 6 and 7). This was due to the large variations in color among seeds of other colors, compared with variation of materially damaged seeds, which showed no or subtle variations. Classifier performance based on the highest probability of occurrence is presented (Tables 8 and 9). Table 8 shows performance of over $58 \%$ for each of the seeds considered. The main misclassifications occurred for pho, with seeds being wrongly predicted as asymptomatic and cer. This did not seem to be the case when all seeds were considered, and there was no instance of pho being classified as $c e r$ (Table 4).

Table 9 shows classification accuracy for symptomatic seeds with highest probability of occurrence. The seeds included damage types from both seeds of other

Table 4. Individual classification accuracy for asymptomatic and symptomatic soybean seeds

\begin{tabular}{|c|c|c|c|c|c|c|c|c|c|}
\hline \multirow[b]{2}{*}{ Seed types ${ }^{a}$} & \multirow[b]{2}{*}{ Quantity } & \multicolumn{8}{|c|}{$\begin{array}{l}\text { Predicted group membership } \\
\text { \% accuracy }\end{array}$} \\
\hline & & asy & $c e r$ & gis & smvbk & alt & fus & pho & smvbr \\
\hline asy & 59 & 58 & 0 & 0 & 0 & 0 & 0 & 1 & 0 \\
\hline$\%$ & & 98.3 & 0 & 0 & 0 & 0 & 0 & 1.7 & 0 \\
\hline cer & 42 & 3 & 35 & 0 & 1 & 1 & 0 & 2 & 0 \\
\hline$\%$ & & 7.1 & 83.3 & 0 & 2.4 & 2.4 & 0 & 4.8 & 0 \\
\hline gis & 44 & 13 & 0 & 30 & 0 & 0 & 0 & 1 & 0 \\
\hline$\%$ & & 29.5 & 0 & 68.2 & 0 & 0 & 0 & 2.3 & 0 \\
\hline$s m v b k$ & 42 & 0 & 1 & 0 & 34 & 5 & 0 & 2 & 0 \\
\hline$\%$ & & 0 & 2.4 & 0 & 81 & 11.9 & 0 & 4.8 & 0 \\
\hline alt & 20 & 12 & 0 & 0 & 0 & 4 & 0 & 4 & 0 \\
\hline$\%$ & & 60 & 0 & 0 & 0 & 20 & 0 & 20 & 0 \\
\hline fus & 24 & 18 & 0 & 0 & 0 & 2 & 0 & 4 & 0 \\
\hline$\%$ & & 75 & 0 & 0 & 0 & 8.3 & $\mathbf{0}$ & 16.7 & 0 \\
\hline pho & 22 & 10 & 0 & 0 & 0 & 2 & 0 & 9 & 0 \\
\hline$\%$ & & 45.5 & 0 & 0 & 0 & 9.1 & 0 & 40.9 & 0 \\
\hline$s m v b r$ & 15 & 1 & 0 & 0 & 0 & 3 & 0 & 1 & 10 \\
\hline$\%$ & & 6.7 & 0 & 0 & 0 & 20 & 0 & 6.7 & 66.7 \\
\hline Overall & 268 & & & & & & & & 67.16 \\
\hline
\end{tabular}

${ }^{\mathrm{a}}$ asy = asymptomatic, cer = Cercospora spp., gis = green immature seed, smvbk = soybean mosaic potyvirus (black), alt $=$ Alternaria spp., fus = Fusarium spp., pho = Phomopsis spp., smvbr = soybean mosaic potyvirus (brown).

Table 5. Classification accuracy for symptomatic soybean seeds only

\begin{tabular}{|c|c|c|c|c|c|c|c|c|}
\hline \multirow[b]{2}{*}{ Seed types $^{\mathrm{a}}$} & \multirow[b]{2}{*}{ Quantity } & \multicolumn{7}{|c|}{$\begin{array}{l}\text { Predicted group membership } \\
\% \text { accuracy }\end{array}$} \\
\hline & & cer & gis & smvbk & alt & fus & pho & smvbr \\
\hline $\begin{array}{l}\text { cer } \\
\%\end{array}$ & 83 & $\begin{array}{l}72 \\
86.7\end{array}$ & 0 & 3 & 2 & 1 & 5 & 0 \\
\hline $\begin{array}{l}\text { gis } \\
\%\end{array}$ & 88 & 0 & $\begin{array}{l}67 \\
76.1\end{array}$ & 1 & 2 & 3 & 15 & 0 \\
\hline $\begin{array}{l}\text { smvbk } \\
\%\end{array}$ & 84 & 3 & 0 & $\begin{array}{l}69 \\
\mathbf{8 2 . 1}\end{array}$ & 8 & 0 & 2 & 2 \\
\hline $\begin{array}{l}\text { alt } \\
\%\end{array}$ & 41 & 0 & 0 & 0 & $\begin{array}{l}27 \\
65.9\end{array}$ & 0 & 11 & 3 \\
\hline $\begin{array}{l}\text { fus } \\
\%\end{array}$ & 47 & 0 & 0 & 0 & 26 & $\begin{array}{l}2 \\
4.3\end{array}$ & 19 & 0 \\
\hline $\begin{array}{l}\text { pho } \\
\%\end{array}$ & 45 & 2 & 0 & 1 & 6 & 0 & $\begin{array}{l}35 \\
\mathbf{7 7 . 8}\end{array}$ & 1 \\
\hline $\begin{array}{l}s m v b r \\
\%\end{array}$ & 29 & 1 & 0 & 0 & 7 & 0 & 0 & $\begin{array}{l}21 \\
72.4\end{array}$ \\
\hline Overall & 417 & & & & & & & 70.3 \\
\hline
\end{tabular}


colors and materially damaged seeds. Most of the misclassification in both categories occurred with smvbk and pho, which showed a close resemblance when cer has a dark purple discoloration.

The occasional instances of asymptomatic seed being misclassified as smvbk, $s m v b r$, alt, or pho can be attributed to dirty or cracked seed coats. Cer was likely to be misclassified due to its occasional dark purple appearance, leading to misclassification as smvbk, and to its occasional light pink appearance, resulting in misclassification as fus. The major misclassification occurred when cer and gis were misclassified as asymptomatic due to their close resemblance. Cer seeds had demonstrated regions on the seed coat unaffected by pink or purple staining (asymptomatic regions), while gis had variation in intensity levels that matched those of asymptomatic, resulting in high misclassification (Table 4). Similarly, fus was excessively misclassified as asymptomatic, alt, and pho.

The misclassifications of alt to smvbr were due to the similarity in brown color. Alt seeds also were misclassified as fus and pho. The tan color of alt was similar to that of some of the seeds infected by fus and pho. Alt was misclassified as fus more than as pho. This was attributed to the similarity of gray color between alt and fus.

Figure 6 shows the scatter plot for the first two canonical discriminant functions for linear discriminant analysis with unequal priors. Asymptomatic, cer, gis, smvbk, and $s m v b r$ formed distinct clusters compared with materially damaged seeds. The variability along the $\mathrm{x}$-axis seems to show classification more toward asymptomatic, cer, gis, alt, and fus; whereas along the yaxis, distinct classification was observed for cer, smvbk, and smvbr. Therefore, the differences among the eight seed types were adequately described primarily by the first function and to a certain level by the second function.

Summary. Linear discriminant analysis with unequal priors was presented in this paper as it reflected the real probabilities of occurrences of asymptomatic and symptomatic seeds. The classification accuracy for symptomatic soybean seeds with highest probability of occurrence ranged from 83 to $93 \%$. The classification accuracy for linear and quadratic functions ranged from 67 to $81 \%$ for test samples with all seed types included. Among the color features, minimum values of RGB contributed to higher classification accuracy in comparison with averages of each of the components. The possible explanation for this contribution was that the RGB averages may have masked the spatial variation. In the case of minimum values, each of the seed types had a minimum level, which was unique.

There was a minimal increase in accuracy when asymptomatic seeds were eliminated compared with all seed types; whereas there was a significant increase in accuracy when seeds of other colors and materially damaged seeds were considered independently of each other and of as-

ymptomatic seeds. When seeds with the highest probability of occurrence were considered, classification accuracy ranged from 83 to $93 \%$. The range of variations

Table 6. Classification accuracy for soybean seeds of other colors (cer, gis, smvbk, and smvbr)

\begin{tabular}{|c|c|c|c|c|c|}
\hline \multirow[b]{2}{*}{ Seed types ${ }^{a}$} & \multirow[b]{2}{*}{ Quantity } & \multicolumn{4}{|c|}{$\begin{array}{l}\text { Predicted group membership } \\
\% \text { accuracy }\end{array}$} \\
\hline & & cer & gis & smvbk & $s m v b r$ \\
\hline $\begin{array}{l}\text { cer } \\
\%\end{array}$ & 83 & $\begin{array}{l}78 \\
94\end{array}$ & 2 & 3 & 0 \\
\hline $\begin{array}{l}\text { gis } \\
\%\end{array}$ & 88 & 0 & $\begin{array}{l}87 \\
98.9\end{array}$ & 1 & 0 \\
\hline $\begin{array}{l}\text { smvbk } \\
\%\end{array}$ & 84 & 2 & 0 & $\begin{array}{l}75 \\
89.3\end{array}$ & 7 \\
\hline $\begin{array}{l}\text { smvbr } \\
\%\end{array}$ & 29 & 0 & 0 & 1 & $\begin{array}{l}28 \\
96.6\end{array}$ \\
\hline Overall & 284 & & & & 94.4 \\
\hline
\end{tabular}

a cer = Cercospora spp., gis = green immature seed, $s m v b k=$ soybean mosaic potyvirus (black), $s m v b r=$ soybean mosaic potyvirus (brown).

Table 7. Classification accuracy for materially damaged soybean seeds

\begin{tabular}{lccccc}
\hline & & \multicolumn{4}{c}{$\begin{array}{c}\text { Predicted group membership } \\
\text { unequal priors \% accuracy }\end{array}$} \\
\cline { 3 - 6 } Seed types $^{\mathbf{a}}$ & Quantity & alt & alt & fus & pho \\
\hline $\begin{array}{l}\text { alt } \\
\%\end{array}$ & 41 & 30 & 31 & 0 & 10 \\
fus & & $\mathbf{7 3 . 2}$ & $\mathbf{7 5 . 6}$ & & 24 \\
$\%$ & 47 & 8 & 23 & 0 & $\mathbf{0}$ \\
pho & & & & 0 & 37 \\
$\%$ & 45 & 9 & 8 & & $\mathbf{8 2 . 2}$ \\
Overall & 133 & & & & $\mathbf{5 1 . 1}$ \\
\hline
\end{tabular}

a alt $=$ Alternaria $\mathrm{spp} .$, fus $=$ Fusarium spp., pho $=$ Phomopsis spp.

Table 8. Classification accuracy for asymptomatic and selected symptomatic soybean seeds with highest probability of occurrence

\begin{tabular}{lcccc}
\hline & & \multicolumn{3}{c}{$\begin{array}{c}\text { Predicted group membership } \\
\text { \% accuracy }\end{array}$} \\
\cline { 3 - 5 } Seed types $^{\mathbf{a}}$ & Quantity & asy & cer & pho \\
\hline asy & 119 & 118 & 0 & 1 \\
$\%$ & & $\mathbf{9 9 . 2}$ & 74 & 3 \\
cer & 83 & 8 & $\mathbf{8 9 . 2}$ & 26 \\
$\%$ & & 16 & 3 & $\mathbf{5 7 . 8}$ \\
pho & 45 & & & $\mathbf{8 8 . 3}$ \\
Overall & & & &
\end{tabular}

a asy $=$ asymptomatic, cer $=$ Cercospora spp., pho $=$ Phomopsis $\mathrm{spp}$.

Table 9. Classification accuracy for selected symptomatic soybean seeds with highest probability of occurrence

\begin{tabular}{|c|c|c|c|c|c|}
\hline \multirow[b]{2}{*}{ Seed types ${ }^{a}$} & \multirow[b]{2}{*}{ Quantity } & \multicolumn{4}{|c|}{$\begin{array}{c}\text { Predicted group membership } \\
\text { unequal priors \% accuracy }\end{array}$} \\
\hline & & cer & $s m v b k$ & alt & pho \\
\hline $\begin{array}{l}\text { cer } \\
\%\end{array}$ & 83 & $\begin{array}{l}74 \\
\mathbf{8 9 . 2}\end{array}$ & 3 & 1 & 5 \\
\hline $\begin{array}{l}s m v b k \\
\%\end{array}$ & 84 & 3 & $\begin{array}{l}70 \\
\mathbf{8 3 . 3}\end{array}$ & 8 & 3 \\
\hline $\begin{array}{l}\text { alt } \\
\%\end{array}$ & 41 & 0 & 0 & $\begin{array}{l}30 \\
73.2\end{array}$ & 11 \\
\hline $\begin{array}{l}\text { pho } \\
\%\end{array}$ & 45 & 1 & 2 & 7 & $\begin{array}{l}35 \\
77.8\end{array}$ \\
\hline Overall & 253 & & & & 82.6 \\
\hline
\end{tabular}

${ }^{a}$ cer $=$ Cercospora spp., gis $=$ green immature seed, smvbk = soybean mosaic potyvirus (black), alt $=$ Alternaria spp., fus $=$ Fusarium spp., pho $=$ Phomopsis spp., smvbr $=$ soybean mosaic potyvirus (brown). 
exhibited within each seed type was expected due to the inclusion of a wide range of seeds comprising crop years 1989 to 1994. In earlier research, the performance of the classifier was improved by replacing seed samples that varied considerably and by treating samples for various years in isolation (5). This approach was not followed in the current research, as the range of variations actually facilitated the development of robust methods for characterization of seed surfaces.

Alt was the dominant source of misclassification, followed by fus and pho, due to its frequent occurrence compared with fus. Asymptomatic seed was the next highest among the categories with misclassification occurrences. Seeds of other colors contributed minimally to misclassification. Among seeds of other colors, smvbr was the main source of misclassification, followed by smvbk and cer. This was attributed to light color variations, which at times may be similar to smvbk and cer. Overall, it was observed that alt was one of the major sources of misclassification, while gis contributed minimally to misclassification for all seed types. The RGB intensity of surface symptoms on seeds varied with disease and within diseases. It was concluded that image processing facilitated the classification of asymptomatic and symptomatic soybean seeds and the development of a knowledge domain based on color information. The performance of the classifier can potentially be enhanced by using a combination of color, morphologi$\mathrm{cal}$, and textural features. The inherent constraints in manual grading and the objectivity of machine vision justify further efforts to automate the grading process.

\section{ACKNOWLEDGMENTS}

This study was supported in part by Hatch Project 10-0323 and Project 55030 of the Illinois Agricultural Experiment Station, College of ACES, University of Illinois at Urbana-Champaign.

\section{LITERATURE CITED}

1. Ahmad, I. S. 1997. Image-based analysis of fungal-damaged soybeans. Ph.D. diss. Department of Agricultural Engineering, University of Illinois at Urbana-Champaign, Urbana.

2. Ahmad, I. S., and Reid, J. F. 1996. Evaluation of color representations for maize images. J. Agric. Eng. Res. 63:185-195.

3. Ahmad, I. S., Reid, J. F., and Paulsen, M. R. 1997. Neuro-fuzzy inference of soybean seed. ASAE Pap. 97-3041. ASAE, St. Joseph, MI.

4. Casady, W. W., and Paulsen, M. R. 1989. An automated kernel positioning device for computer vision analysis of grain. Trans. ASAE 33:1821-1826.

5. Casady, W. W., Paulsen, M. R., Reid, J. F., and Sinclair, J. B. 1992. A trainable algorithm for inspection of soybean seed quality. Trans. ASAE 35:2027-2034.

6. CIE. 1989. International Lighting Vocabulary. CIE Publ. 17.4. Central Bureau of the Commission Internationale de L'Eclairage, Vienna, Austria.

7. Cooke, R. A., Mostaghimi, S., and Woeste, F. E. 1993. VTFIT: A microcomputer-based routine for fitting probability distribution func- tions to data. Appl. Eng. Agric. 9:401-408.

8. Doupnik, B., Jr. 1993. Soybean production and disease loss estimates for north central United States from 1989 to 1991. Plant Dis. 77:1170-1171.

9. GIPSA-FGIS. 1994. Grain Inspection Handbook. Book II, Soybeans. Grain Inspection Packers, Stockyards Administration-Federal Grain Inspection Service, U.S. Dep. Agric.

10. Gunasekaran, S., Cooper, T. M., and Berlage, A. G. 1988. Evaluating quality factors of corn and soybeans using a computer vision system Trans. ASAE 31:1264-1271.

11. Manly, B. 1986. Multivariate Statistical Methods: A Primer. Chapman and Hall, New York.

12. Marks, S., and Dunn, O. J. 1974. Discriminant functions when covariance matrices are unequal. J. Am. Stat. Assoc. 69:555-559.

13. Mbuvi, S. W., Litchfield, J. B., and Sinclair, J. B. 1989. Physical properties of soybean seeds damaged by fungi and a virus. Trans. ASAE 32:2093-2096.

14. Paulsen, M. R., Wigger, W. D., Litchfield, J. B., and Sinclair, J. B. 1989. Computer image analyses for detection of maize and soybean kernel quality factors. J. Agric. Eng. Res. 43:93-101.

15. Russ, J. C. 1995. The Image Processing Handbook. 2nd ed. CRC Press, Boca Raton, FL.

16. Sinclair, J. B. 1992. Discoloration of soybean seeds-An indicator of quality. Plant Dis. 76:1087-1091.

17. Sinclair, J. B. 1993. Phomopsis seed decay of soybeans-A prototype for studying seed disease. Plant Dis. 77:329-334

18. Sinclair, J. B., and Backman, P. A. 1989. Compendium of Soybean Diseases. 3rd ed. American Phytopathological Society, St. Paul, MN.

19. Zayas, I. Y., and Steele, J. L. 1996. Image texture analysis for discrimination of mill fractions of hard and soft wheat. Cereal Chem. 73:136-142. 